\title{
EN RESPUESTA AL COMENTARIO DE CARLOS PEREDA SOBRE UN LUGAR PARA LA MORAL
}

\author{
JoSEP CORBí \\ Facultad de Filosofía y Ciencias de la Educación \\ Universidad de Valencia \\ Josep.Corbi@uv.es
}

RESUMEn: Carlos Pereda califica mi concepción de la moral de realismo particularista y objeta a mi defensa tanto del realismo como del particularismo. En mi respuesta trato de mostrar cómo nuestras discrepancias en torno al papel de los principios en la deliberación moral es, excepto en un punto crucial, cuestión de énfasis. No ocurre lo mismo, sin embargo, con mi reivindicación del realismo moral, pues parte de lo que intento mostrar en el libro es que los programas constructivistas de los que habla Pereda no pueden pensarse coherentemente.

PALABRAS CLAVE: principios, deliberación moral, realismo moral, particularismo moral

SUMMARY: Carlos Pereda presents my view about morality as a sort of particularist realism and objects both to my defence of realism and that of particularism. In my reply, I argue that our discrepancies about the role of principles in moral deliberation is, except in a crucial respect, a matter of emphasis. Something quite different happens, however, with my vindication of moral realism. For part of what I try to show in my book is that constructivist programs like the one suggested by Pereda cannot be coherently thought.

KEY WORDS: principles, moral deliberation, moral realism, moral particularism

1. Es cierto que, como señala Carlos Pereda, Un lugar para la moral tiene algo de novela de formación, de novela en la que el autor reconstruye su proceso de formación en la confianza de que su recorrido no sea ajeno al de sus lectores, sino que, por el contrario, contribuya a que éste defina con mayor nitidez los perfiles de su propio proceso o, incluso, descubra hitos que le permitan orientarse donde se sentía perdido. A pesar de la abundancia de elementos narrativos que subrayan el carácter novelesco de ese proceso formativo, el foco de atención es conceptual. Se trata de contar historias que ayuden al lector a 
identificar y reconstruir los hilos argumentativos que articulan la manera en que, desde la modernidad, concebimos nuestro lugar en el mundo. Por eso me parece acertado que Pereda nos invite a leer este ensayo como "una novela de aprendizaje conceptual".

2. El proceso de aprendizaje parte de la visión del mundo que desarrollan las ciencias naturales y del reto que ella supone para nuestra capacidad de responder a la pregunta "¿Cómo debo vivir?" Parece que, desde el punto de vista de las ciencias naturales, el mundo está desencantado, nada es bueno ni malo, feo o hermoso, y que todo valor, ya sea moral o estético, ha de derivar de nuestra subjetividad, de cuáles sean nuestros deseos e intereses. Nos encontramos, de este modo, ante lo que Pereda denomina el dilema moderno:

O renunciamos a la concepción científica del mundo tradicionalmente aceptada — la visión "desencantada" de la naturaleza- y su manera de concebir lo objetivo y lo subjetivo; o reducimos los juicios morales a los gustos y los apetitos arbitrarios de cada individuo. (Pereda 2004, p. 68)

Este dilema constituye, ciertamente, el punto de partida del libro y coincido con Pereda en que, en el fondo, se trata de un pseudodilema. De hecho, uno de los propósitos centrales del libro consiste justamente en mostrar cómo la mosca puede salir de la botella y, en concreto, por qué el dilema en el que parece que estamos atrapados deriva de una mala comprensión de lo que la ciencia nos enseña acerca del mundo, así como de las condiciones que fijan el contenido de nuestros juicios morales.

3. Entiende Pereda, sin embargo, que mi manera de escapar del dilema no resulta del todo satisfactoria y que ello se debe, en parte, a que hago una "lectura quizá poco productiva de la tradición kantiana" y, específicamente, de su manera de concebir el papel de los principios universales en la deliberación moral. En concreto, Pereda califica mi propuesta acerca del lugar que la moral pueda ocupar en el mundo de realismo particularista y 
plantea objeciones a mi defensa tanto del realismo como del particularismo. En los párrafos que siguen trataré de mostrar cómo nuestras discrepancias en torno al papel de los principios en la deliberación moral es más bien una cuestión de énfasis, excepto en un punto que tal vez se revele crucial. No ocurre lo mismo, empero, con mi reivindicación del realismo moral, pues parte de lo que intento mostrar es que los programas constructivistas de los que habla Pereda y que pretenden aislar el mundo de la razón teórica del mundo de la razón práctica, el mundo sensible del inteligible, no pueden pensarse coherentemente; esto se debe a que ni siquiera pueden llegar a fijar el contenido (no ya su validez o invalidez) de los juicios morales sin atribuir propiedades morales al mundo sensible. Empecemos, entonces, por las sospechas que Pereda lanza en contra de mi defensa del realismo moral.

4. En el libro pretendo mostrar que el subjetivismo moral - la idea de que los rasgos morales de las acciones son simplemente proyecciones de ciertos estados subjetivos - no puede pensarse coherentemente, que los sentimientos morales están sometidos a una disciplina ausente en los gustos y deseos. Puedo sentir repugnancia ante cualquier objeto, por más extraño o ridículo que parezca, pero no puedo sentir repugnancia moral más que ante ciertos objetos o, al menos, ante objetos que están vinculados con ciertos rasgos moralmente relevantes. La repugnancia que uno siente ante la contemplación de los horrores de Auschwitz no puede ser más que moral; mientras que la repugnancia que a uno le pueden provocar las tripas de un pollo no es, en principio, una repugnancia moral a no ser que conectemos esas tripas con ciertos rasgos moralmente relevantes; por ejemplo, las condiciones en las que esos pollos se crían y mueren.

Considera Pereda, no obstante, que el término "proyección" unifica indebidamente las propuestas humeanas o neohumeanas con las kantianas y que podemos retener la disciplina que reconozco en los sentimientos morales sin comprometernos con el realismo moral, sin borrar la diferencia entre la razón teórica y la razón práctica. Pereda defiende que podemos descubrir la necesidad propia de los juicios morales, y que los diferencia de 
los juicios de gusto, en el proceso de reflexión que nos lleva de lo universal a lo particular y viceversa:

En alguna medida, todas y todos reafirmamos o negamos reflexivamente nuestras necesidades, deseos y emociones incluso aquellos en apariencia "más naturales". Ese proceso de reafirmación o de negación, ese proceso de argumentación con los otros y consigo mismo, reitero, se conforma tanto por instancias universales como por particulares y se lleva a cabo, de manera implícita y, en algunas ocasiones, explícitamente, a lo largo de cada vida. (Pereda 2004, p. 73)

Y en ello estriba el núcleo del programa constructivista de Pereda, en el que los principios desempeñan un papel central, pues no hay rasgos morales en el mundo que determinen lo moralmente correcto o incorrecto; más bien, la evaluación moral de una situación ha de surgir de su examen a la luz de ciertos principios generales cuyo fundamento no puede descansar en la atribución de rasgos morales al mundo. Y esta necesidad proporciona un vínculo argumentativo entre su rechazo del realismo moral y su descrédito del particularismo.

5. No creo, sin embargo, que las observaciones que se recogen en la cita de Pereda afecten a mis argumentos en contra de la posibilidad de fijar el contenido de los juicios morales, ya sean particulares o universales, sin atribuir rasgos morales al mundo sensible. En esa cita se describe el proceso de equilibrio reflexivo mediante el que evaluamos tanto nuestros juicios particulares como nuestros principios, pero ese proceso presupone que tales juicios y principios tienen ya un contenido cuya corrección se evalúa. Y mi crítica del subjetivismo moral se centra precisamente en ese presupuesto, es decir, en las condiciones en las que su contenido puede fijarse y no en cómo evaluamos los juicios una vez que hemos fijado su contenido. En este sentido, considero que las observaciones de Pereda no aportan mucha luz acerca de cómo podría fijarse el contenido de los juicios morales sin comprometerse con el realismo. El ir y venir de lo general a lo particular puede ser uno de los elementos del 
círculo hermenéutico en el que se fija su contenido, pero no resuelve por sí mismo el problema que planteaba al inicio de la sección 4: identificar las respuestas morales en función de los rasgos de los objetos que las merecen. Es cierto que Pereda es poco explícito en este punto de su comentario y que seguramente su programa constructivista contará con propuestas para fijar ese contenido sin adquirir un compromiso realista. Puedo añadir en defensa de mi posición que, en Corbí (2004), intento mostrar cómo fracasan los dos intentos más prometedores (a saber, el disposicionalismo moral y el realismo procedimentalista) de fijar ese contenido sin atribuir rasgos morales al mundo. Esto me conduce a concluir que los programas constructivistas no pueden llevarse a cabo coherentemente, porque no pueden, por un lado, conservar la visión desencantada del mundo y, por otro, distinguir la necesidad moral de la variabilidad de los juicios de gusto y los deseos.

Ahora bien, más que ayudarnos a escapar del dilema moderno, esta conclusión parece sumirnos más profundamente en él: la reducción de los juicios morales a los gustos y apetitos de cada individuo no sólo nos resulta moralmente inquietante, sino que, según acabo de argumentar, no podemos llevarla a cabo coherentemente. Mi manera de intentar sacar la mosca de la botella consiste, no obstante, en poner en tela de juicio el otro extremo del dilema, a saber, la visión desencantada del mundo.

La noción de causa completa yace en el fondo de la visión mecanicista (y, por tanto, desencantada) del mundo. De acuerdo con esa noción, cada suceso físico tiene una causa física completa, es decir, un conjunto de condiciones físicas antecedentes de las que, junto con las leyes de la naturaleza, se sigue inexorablemente el suceso físico en cuestión. En Corbí y Prades (2000), argumentamos con detenimiento que la noción de causa completa es internamente incoherente porque: (a) no hay ningún conjunto de condiciones físicas antecedentes del que, junto a las leyes de la naturaleza, se siga inexorablemente (es decir, en todos los mundos físicamente posibles) un efecto físico, pues es imprescindible añadir la cláusula "y, en ese contexto, ésas son todas las condiciones físicas involucradas" y eso conlleva un 
anclaje a un contexto causal que es justamente lo que la noción de causa completa pretendía eliminar; (b) aun si resolviésemos esta dificultad y pudiésemos enumerar tales condiciones físicas antecedentes, eso nos obligaría a entrar en un nivel de detalle tal que, para cada suceso físico que propusiésemos, siempre podríamos encontrar que se da más de un conjunto de condiciones que garantiza inexorablemente la producción del efecto, por lo que la identificación de causas completas supone que todos los procesos causales están sobredeterminados y eso es algo que ningún mecanicista está dispuesto a aceptar.

En el capítulo 8 de Un moral lugar para la moral, encontramos una breve reconstrucción de estas dos líneas argumentativas, mientras que en el capítulo 9 intento extraer las consecuencias que se pueden derivar para la objetividad de la moral. El hecho de que la causa de un suceso físico sólo pueda identificarse sobre un trasfondo causal, y que la distinción entre causa y trasfondo causal dependa ineludiblemente de los intereses que guían la investigación, me lleva a concluir que la objetividad de los procesos causales que descubre la ciencia no puede consistir en que tales procesos tengan condiciones de identidad totalmente independientes de nuestras prácticas de investigación, que si queremos reconocer los fenómenos físicos como objetivos, hemos de apelar a otra noción de objetividad, una noción que haga compatible la objetividad de un fenómeno con el hecho de que sus condiciones de identidad no sean independientes de nuestras prácticas.

Todavía se podría argumentar, sin embargo, que el modo en que las propiedades morales dependen de nuestros intereses difiere sustancialmente de cualquier sentido en el que la identificación de los procesos causales sea relativa a los intereses que guían nuestras prácticas investigadoras. En este punto examino los dos argumentos que considero más poderosos en favor de esa diferencia: el argumento del acuerdo y el argumento del éxito. En ambos casos concluyo que tales argumentos sólo ayudan a trazar la diferencia entre las propiedades físicas y las morales si presuponemos la noción de causa completa, la concepción desencantada del mundo, cuya incoherencia interna estábamos 
dando por supuesta en este momento de la discusión. Por eso concluyo diciendo:

No cabe duda de que, para cualquier estándar razonable de objetividad que se pueda elaborar, las teorías científicas podrán satisfacerlo. El propósito principal de este libro ha consistido precisamente en mostrar que, en cualquier sentido en el que podamos decir legítimamente que la ciencia nos revela aspectos objetivos del mundo, deberemos reconocer que en ese mundo hay también hechos morales como el engaño o la tortura. (Corbí 2003, p. 178)

Una vez establecido el sentido en el que defiendo la objetividad de la moral y por qué pienso que los programas constructivistas no pueden dar cuenta del contenido de nuestros juicios morales, podemos pasar a examinar las razones por las que Pereda piensa que no atribuyo a los principios el papel que merecen en la deliberación moral.

6. Como ya dije, mis discrepancias con Pereda respecto al papel de los principios en la deliberación moral son fundamentalmente cuestión de énfasis, excepto en un punto crucial que en su momento destacaré.

En Un lugar para la moral elaboro con tanto detalle los argumentos que ponen de manifiesto los límites del papel que los principios generales desempeñan en la deliberación moral, que uno puede fácilmente pensar que mi propósito es mostrar que los principios no cumplen ningún papel en ese modo de deliberación. Sin embargo, nada más lejos de mi intención; de hecho, reconozco explícitamente en mi ensayo cada uno de los papeles que Pereda atribuye a los principios. Básicamente, Pereda entiende que (a) los principios universales son "principios con los cuales no sólo nos autodeterminamos sino que nos autoconstituimos en la persona que somos" (Pereda 2004, p. 70); pero (b) insiste en que los principios universales están subdeterminados y que se requieren otros elementos para fijar la respuesta a preguntas prácticas particulares. En el juego de lo general a lo particular vamos formándonos como sujetos y vamos definiendo las respuestas correctas a cada cuestión moral: 
Una vez que hayamos cultivado tales virtudes, probaremos si el ejercicio de tales virtudes conduce a la realización del principio en cuestión y, a su vez, los principios, en cuanto propuestas subdeterminadas, se irán determinando en un ir y venir recíproco y sin fin. (Pereda 2004, p. 71)

Respecto a (a), he de decir que, en Un lugar para la moral, no sólo reconozco el papel del imperativo categórico en la deliberación moral una vez que éste se entiende en términos no formales, es decir, como un imperativo que se alimenta de intuiciones morales particulares; sino que, siguiendo a Alasdair MacIntyre, ${ }^{l}$ subrayo el papel de los fines generales (y, por tanto, de los principios) en la deliberación práctica:

Podemos entender la unidad de la vida de una persona reflexiva a partir del concepto medieval de búsqueda, un concepto que recoge dos rasgos cruciales de la deliberación constitutiva. Es necesario para esa búsqueda que haya un telos, una finalidad, mínimamente definida que oriente la deliberación. Se perfila así uno de los puntos de partida de la reflexión que contribuye a articular la vida de una persona y en cuyos términos esa persona evaluará retrospectivamente su propia existencia. Pero es todavía más difícil determinar, en cada caso, en qué consiste ser fiel a ese telos, y es en este punto donde la búsqueda alcanza su verdadera dificultad y se halla plagada de incertidumbres. No hay receta alguna que sustituya al doloroso proceso de la búsqueda, de la deliberación constitutiva. Esa búsqueda es, a un tiempo, el modo en el que cada persona va articulando la red de significaciones que constituye su vida, y la perspectiva desde la que evalúa el valor de la misma, su capacidad de ser fiel a lo importante, a lo que merece la pena. (Corbí 2003, p. 129)

El papel que atribuyo en este texto al telos está, a mi entender, íntimamente emparentado con el papel de los principios universales en la autoconstitución del sujeto práctico que Pereda destaca en (a). Por otro lado, las dificultades para definir, en cada circunstancia, en qué consiste ser fiel a ese telos no son

${ }^{1}$ Cfr. MacIntyre 1981, cap. 15. 
tampoco ajenas al ir y venir entre lo general y lo particular que se recoge en (b).

7. Coincido igualmente con Pereda en que una de las lecciones que podemos aprender del fenómeno de la suerte moral es que, a la hora de imputar responsabilidades, hemos de evitar tanto el vértigo simplificador, que se desentiende totalmente de las consecuencias de nuestros actos, como el vértigo complicador, que nos convierte en responsables de todas las consecuencias de nuestros actos. Es ésta, a mi juicio, otra manera de aludir al hecho de que, en la imputación de responsabilidades, hemos de atender al margen de maniobra que el sujeto tenga en la situación de la que se trate y que la aplicación estricta del principio según el cual sólo somos responsables de lo que cae bajo nuestro control es de escasa utilidad. Es a esta idea precisamente a lo que aludo en la cita que Pereda presenta como expresión más clara de mi descrédito de los principios: "los principios generales no nos ayudan en exceso a la hora de determinar si somos o no responsables de ciertos sucesos" (Corbí 2003, p. 94), pues, la cita continúa: "Esos principios generales aludían, como vimos, a que uno sólo es responsable de lo que cae bajo su control y a que uno es responsable de lo que hace" (p. 95). Es decir, en este punto no estoy negando el papel de los principios generales en la deliberación práctica, sino mostrando cómo los mejores principios que se han esgrimido para delimitar nuestras responsabilidades no son útiles si no vienen mediados por cierto sentido del margen de maniobra que los principios mismos no pueden ofrecer. Y creo que, en esta observación, recojo la misma idea que Pereda expresa cuando nos advierte acerca de la necesidad de evitar tanto el vértigo simplificador como el complicador.

8. Hay, sin embargo, un punto donde se manifiesta nuestra discrepancia fundamental y que, como veremos, fija un limite muy significativo a lo que los principios pueden aportar a la deliberación práctica y que Pereda parece negar. Así, en un momento de sus comentarios, Pereda destaca: 
Respecto del principio de no tratar a ninguna persona como mero medio, se enfatizará su carácter de prohibición absoluta indicando que nunca, ni siquiera frente a la causa que consideremos más sagrada, o más útil para nuestra comunidad, y hasta para la humanidad en su conjunto, podremos tener esclavos o ser racistas, podremos poner una bomba en un tren o en una escuela. (Pereda 2004, p. 71; las cursivas son mías.)

Este texto parece excluir la posibilidad de que los agentes morales nos encontremos ante un genuino dilema moral, como el dilema al que se enfrentaron quienes finalmente decidieron poner una bomba en un transbordador, a sabiendas de que morirían personas inocentes, para evitar que Hitler trasladase su arsenal de agua pesada desde Noruega hasta Alemania con el fin de fabricar la bomba atómica. Es difícil pensar que, en ese caso y tomadas las cautelas adecuadas para reducir al máximo el costo humano, no estuviese justificado poner una bomba en el transbordador, aunque, ciertamente, ese "nunca" que Pereda menciona siga reverberando en nuestro pesar por las muertes que acompañaron a la acción.

Lo que defiendo, en Un lugar para la moral, es precisamente que existen muchos casos de conflictos de valores, de dilemas morales, en los que no hay principio alguno que nos indique cómo actuar, qué hacer, y que, en ese punto de la deliberación, hemos de acudir inexorablemente al juicio del hombre prudente, juicio que, a su vez, no puede descansar en último término en principios, sino en la visión de una persona bien formada, con la sensibilidad adecuada. Éste es el sentido en el que podemos entender que el tribunal último de lo correcto o de lo incorrecto no son los principios, sino el juicio del hombre prudente, su percepción de un asunto particular. Naturalmente, ese juicio no es un juicio desnudo, puede arroparse de razones, de principios, que de alguna manera lo ayudan a ver los aspectos relevantes de la situación, pero el criterio último no pueden ser los principios, pues éstos entran en conflicto los unos con los otros sin que exista principio alguno que pueda mediar entre ellos, sino la percepción. Se sigue de ello que el ideal del deliberador competente no será ya el de una persona que deja de 
lado sus pasiones y aplica meticulosamente los principios de la razón, sino el de alguien que, fruto de una buena formación de su carácter, percibe con sutileza y corrección el modo en que ciertas experiencias se conectan con otras. Y, como hemos visto, en la formación de esa persona los principios desempeñarán un papel relevante, pero no podremos ya reconocerlos como el tribunal último de lo moralmente correcto o incorrecto.

Esta insistencia en la percepción, en ver lo que hay, casa suavemente con el realismo moral que defiendo y tiene más problemas para integrarse en los programas constructivistas que Pereda propone. En cualquier caso, y como él mismo lo indica, éste es sólo un paso más en una discusión honesta y apasionada por lo que más nos importa: nuestra humanidad. ${ }^{2}$

\section{BIBLIOGRAFÍA}

Corbí, J.E., 2004, "Normativity, Moral Realism, and Unmasking Explanations", Theoria, vol. 19/2, no. 50, pp. 155-172.

, 2003, Un lugar para la moral, Antonio Machado Editores, Madrid.

Corbí, J.E. y J.L. Prades, 2000, Minds, Causes, and Mechanisms. A Case against Physicalism, Blackwell, Oxford.

MacIntyre, A., 1981, After Virtue, University of Notre Dame Press, Notre Dame.

Pereda, C., 2004, “¿Puede la moral prescindir de principios universales? Una discusión con Josep Corbí”, Crítica (en este mismo número, pp. 67-74).

Recibido el 15 de noviembre de 2004; aceptado el 1 de diciembre de 2004.

\footnotetext{
${ }^{2}$ Quiero, en primer lugar, agradecerle a Marta Moreno sus comentarios acerca de la primera versión de este escrito y señalar, en segundo término, que éste se ha elaborado en el seno del proyecto de investigación "Creencia Motivación y Verdad", parcialmente subvencionado por la Generalitat Valenciana (GV04B-251 y GRUPOS04/48) y por el Ministerio de Ciencia y Tecnología (BFF2003-08335-C03-01).
} 